\section{Gravidez na adolescência, pré-natal e resultados perinatais em Montes Claros, Minas Gerais, Brasil}

\author{
Adolescent pregnancy, prenatal care, \\ and perinatal outcomes in Montes Claros, \\ Minas Gerais, Brazil
}

\footnotetext{
1 Escola Paulista de Medicina, Universidade Federal de São Paulo, São Paulo, Brasil.

2 Departamento de Saúde da Mulher, Universidade Estadual de Montes Claros, Montes Claros, Brasil.

Correspondência P. Goldenberg Departamento de Medicina Preventiva, Escola Paulista de Medicina, Universidade Federal de São Paulo. Rua Pedro Toledo 675, São Paulo, SP 04039-032, Brasil. paulete@medprev.epm.br
}

\begin{abstract}
The aim of the present study was to measure the proportion of adolescent pregnancies in Montes Claros, Minas Gerais, Brazil. From a total of 7,672 live births in 2001, the estimated proportion of births by adolescent mothers was $21.5 \%$. In addition to the limited presence of adequate frequency in prenatal visits, especially in the 1014-year bracket (12.0\%), the study identified an increase in the number of complications, inversely related to age, and these differences were significant in relation to prematurity and low birth weight, which did not occur with the 5minute Apgar score. Confirming the hypothesis of greater frequency of these complications when the number of prenatal visits was inadequate, the outcomes also signal an age-associated risk, particularly in early adolescence (10-14 years). These results, together with the data on an increase in adolescent pregnancy in the region from 1997 to 2001, point to teenage pregnancy as a public health problem.
\end{abstract}

Pregnancy in Adolescence; Prenatal Care; Gestation Age; Low Birth Weight Infant; Apgar Score
Paulete Goldenberg ${ }^{1}$

Maria do Carmo Tolentino Figueiredo 2 Rebeca de Souza e Silva 1

\section{Introdução}

A adolescência é o período que se caracteriza pela transição da infância para a idade adulta, ou seja, pela perda da identidade infantil, busca da identidade adulta, sendo, assim, uma fase de profunda instabilidade emocional e mudanças corporais 1 . Considerando que a adolescência é social e historicamente modelada, tal afirmação nos remete à perspectiva de sua significação no mundo contemporâneo ocidental.

Restringindo a questão ao plano reprodutivo, de acordo com Beretta et al. 2, a maioria destes jovens, em nosso meio, chega à maturidade sexual antes de atingir a maturidade social, emocional ou a independência econômica. Dentre múltiplas determinações, a erotização do adolescente, promovida pela mídia, segundo aponta Fujimori et al. ${ }^{3}$, estimula a iniciação sexual precoce que, na ausência do domínio das práticas contraceptivas, pode resultar em gravidez não desejada.

Mais freqüente nos segmentos sociais mais desfavorecidos, a gravidez na adolescência representa, em significativo número de casos, um agravante no complexo quadro existencial, comprometendo o futuro profissional, dificultando o retorno à escola e limitando as oportunidades de trabalho 3,4,5,6,7,8. A propósito, mesmo lembrando que nem toda gravidez na adolescência é indesejada, como aponta Daadorian ${ }^{9}$, não se pode deixar de disponibilizar ser- 
viços assistenciais condizentes e orientações que facultem práticas contraceptivas responsáveis no exercício da sexualidade para esse segmento social. Em meio à diversidade e complexidade de injunções associadas à ocorrência da gravidez na adolescência, seu enfrentamento configura, certamente, um desafio à sociedade.

Ao lado das potenciais repercussões, no plano existencial, associadas à gravidez na adolescência, há indícios, no plano biológico-social, de maior concentração de agravos à saúde materna, bem como de complicações perinatais, particularmente entre adolescentes mais jovens. Dentre as complicações maternas e neonatais mais freqüentes da gravidez na adolescência, são referenciados o baixo ganho de peso materno, a desproporção céfalo-pélvica, a préeclampsia, a prematuridade, o baixo peso ao nascer e o Apgar baixo no quinto minuto. $\mathrm{O}$ baixo peso ao nascer é o mais importante fator associado à mortalidade e morbidade perinatais, segundo a Organização Mundial de Saúde (OMS) 10 , quando se avalia o desfecho de uma gravidez. Segue, em importância, a prematuridade considerada a idade gestacional abaixo de 37 semanas - na medida em que pode predispor a problemas imediatos ao nascimento ou tardios, tais como: hipóxia, síndrome da membrana hialina, tocotraumatismos, hemorragias intracranianas, infecções, hipoglicemia e atraso no desenvolvimento neuropsicomotor futuro. O Apgar no quinto minuto, referindo o estado de oxigenação do recém-nascido no período ante e intraparto, é considerado importante preditor da avaliação do bem-estar e do prognóstico inicial do recém-nascido, sinalizando para um bom estado deste a partir de valores acima de 7, como refere Correa 11.

Hábitos como o uso de álcool, drogas e o fumo, doenças sexualmente transmissíveis, anemia, desnutrição, abuso sexual e físico podem, com freqüência, aumentar a morbidade para a mãe e para o feto 4,12 . Vários autores, questionando a importância estrita da idade, ressaltam que as complicações relativas aos desfechos da gravidez na adolescência se associam às condições sociais de existência, relacionadas com o nível de escolaridade, estado civil, apoio familiar e, sobretudo, com um adequado acompanhamento de pré-natal 12,13,14,15,16. A propósito, o número condizente de consultas de pré-natal, referenciado a um adequado acompanhamento da gestação, vem sendo apontado como condição interveniente na incidência de complicações na adolescência 7,17,18,19,20.

A questão da gravidez na adolescência e complicações perinatais que encerra, no contexto das complexas injunções sociais de sua ocorrência, tem seu espectro amplificado em meio às profundas transformações nos padrões reprodutivos e demográficos em curso no Brasil. Desde meados dos anos 70, registrase, no país, uma abrupta queda da taxa de fecundidade total, que passa de 5,76 filhos por mulher em 1970 para 2,70 em 1990, traduzindo um progressivo rejuvenescimento da estrutura etária da fecundidade 21. Em consonância com esse processo, as taxas de fecundidade específicas por idade experimentam generalizada queda, excetuando-se o segmento de 15 a 19 anos de idade. A participação dessas jovens na fecundidade total aumenta, passando de $7,1 \%$ em 1970 para 9,1\% em 1980 e 13,5\% em 1990. Melo 21 salienta, ainda, que, ao lado dessa participação proporcional crescente entre as jovens de 15 a 19 anos, as regiões do país, com exceção da região Sul, viram crescer as taxas específicas de fecundidade no período dos anos 70 aos 90 . Nesse contexto, inscreve-se o aumento do número de filhos de mães adolescentes, agravado pela presença de mães de 10 a 14 anos de idade.

Focalizando a ocorrência de gestações em 457 pacientes de baixa renda com idade entre 12 a 43 anos, Monteiro \& Cunha 22 identificaram uma freqüência de $13,6 \%$ em adolescentes abaixo de 16 anos e de 40,0\% quando a idade era inferior a 19 anos, em serviço público no Rio de Janeiro. Motta \& Pinto e Silva 14 também observaram aumento de $12,2 \%$ dos partos ocorridos entre adolescentes com idade abaixo de 16 anos no período de 1977 a 1990, apontando para sua ocorrência inclusive na adolescência precoce.

Nesse contexto, emerge e reverbera o reconhecimento da questão da gravidez na adolescência como crescente problema de saúde pública em nosso meio. Constituindo um desafio, o enfrentamento da atenção à gravidez na adolescência se impõe em Montes Claros. Sediada na região norte de Minas Gerais, com um Índice de Desenvolvimento Humano (IDH) próximo aos valores mais baixos em âmbito nacional, a cidade, em acelerado processo de urbanização, comporta o segundo entroncamento rodoviário do país. Constituindo um centro universitário e assistencial, Montes Claros se institui como rede de referência regional, inclusive para atendimento de gravidez de alto risco.

Consubstanciando a preocupação com a presença significativa de gravidez na adolescência na rede hospitalar de Montes Claros, vivenciada no quotidiano dos serviços, observou-se, no período de 1994 a 1996, um aumento na proporção de nascidos vivos nesse segmento etário, que passou de $9,06 \%$ para $17,57 \%$ 
do total de partos. Em consonância com recomendações de agências nacionais e internacionais 23 , entrou em funcionamento, em 1999, um serviço especializado de assistência, voltado para o atendimento ao adolescente, na policlínica da Universidade Estadual de Montes Claros.

Paralelamente ao desafio do enfrentamento que se coloca, a implantação de um sistema de informatização dos dados vitais na região, plenamente instituído no ano 2000, permitiria monitorar a ocorrência de gravidez na adolescência, por referência às adultas, atendendo a indagações presentes sobre a extensão e implicações de sua ocorrência na rede hospitalar da cidade.

Nesse sentido, o estudo propõe, em primeira instância, estimar o coeficiente específico de fecundidade entre adolescentes de 10 a 14 anos de idade e de 15 a 19 anos, ao lado da proporção de mães assistidas na rede hospitalar de Montes Claros, considerando a hipótese que esses valores, resguardadas as especificidades regionais, aproximariam-se aos do país. Em segunda instância, trata-se de caracterizar o perfil das parturientes, adolescentes e adultas, segundo a freqüência ao pré-natal e tipo de parto, assim como em função das condições dos nascidos vivos, relativas à idade gestacional, peso ao nascer e Apgar do quinto minuto - considerando a hipótese de que as adolescentes, sobretudo as mais jovens, concentram maiores proporções de nascidos vivos com resultados adversos - prematuridade, baixo peso e Apgar no quinto minuto abaixo de 7 - na ausência de adequada proteção assistencial.

\section{Metodologia}

O presente levantamento envolveu o dimensionamento do número de nascidos vivos ocorridos em Montes Claros, a partir do Sistema de Informações sobre Nascidos Vivos (SINASC), relativo ao ano de 2001.

Tendo em vista sua ocorrência entre adolescentes, foram calculadas duas medidas: a proporção de nascidos vivos de mães adolescentes em relação ao total de mulheres que deram à luz a um nascido vivo na rede hospitalar da cidade, bem como a taxa específica de fecundidade na adolescência (número de nascidos vivos de mães adolescentes residentes em Montes Claros dividido pela população de mulheres dessa faixa etária do município por cem mulheres). Para efeito de comparação, as mesmas medidas foram calculadas para o país, com base nos dados do DATASUS/Censo de 2000 (Instituto Brasileiro de Geografia e Estatística.
Censo demográfico 1991-2000. http:/ / www.ibge. br, acessado em 25/Mar/2003), referenciadas a 1997, tendo em vista situá-las no espaço temporal dos últimos cinco anos.

$\mathrm{Na}$ caracterização das parturientes e respectivos desfechos, de um total de 7.852 Declarações de Nascidos Vivos, foram excluídas 176 gemelares e 4 nas quais não constava o registro de idade, restando, no estudo, por conseguinte, 7.672 Declarações de Nascidos Vivos.

Obedecendo aos critérios estabelecidos pela OMS 10, foram consideradas adolescentes as mães com idade entre 10 e 19 anos - distinguindo-se a adolescência precoce (10 a 14 anos) da tardia (15 a 19 anos) - e adulta, a parturiente com idade igual ou superior a 20 anos. Das 7.672 Declarações de Nascidos Vivos que integraram o estudo, 51 eram de mães de 10 a 14 anos de idade, 1.605 , de 15 a 19 anos, e 6.016 tinham 20 anos e mais. Vale dizer, no caso da caracterização das parturientes e nascidos vivos, que foram diversificados os totais por variáveis em função de perdas distintas devidas à ausência de informação ou registro de "informação ignorada".

Para cada uma dessas faixas etárias, foram considerados a realização de pré-natal e o tipo de parto. Em relação ao pré-natal, sob inspiração das recomendações do Ministério da Saúde 11 , adaptadas às condições de registro de freqüência a esses serviços nas Declarações de Nascidos Vivos (que, no ano de 2001, era realizado por intervalos de classe: 0 a 3,4 a 6 e 7 e mais), foram adotas as seguintes categorias: pré-natal adequado, quando a gestante compareceu em mais de seis consultas; pré-natal não-adequado, quando a gestante compareceu em até seis consultas ou não realizou pré-natal. Os tipos de parto foram classificados como: cesárea ou não-cesárea.

No tocante às condições dos nascidos vivos, em relação à variável idade gestacional, os dados foram agrupados em recém-nascido prematuro, aquele com idade gestacional abaixo de 37 semanas; e não prematuro, os que nasceram a termo (entre 37 a 41 semanas) e pós-termo (com idade gestacional de 42 e mais semanas de gestação). O peso ao nascer foi classificado como sendo de baixo peso para os nascituros com até 2.499 gramas. No tocante ao Apgar no quinto minuto, foram consideradas as categorias: abaixo de 7 e igual ou superior a 7 .

Para efeito de comparação das variáveis em estudo nos distintos segmentos etários, foram calculados os intervalos de confiança de $95 \%$.

Levando em conta a relevância do pré-natal e do tipo de parto na consideração das associações entre idade da mãe e as condições dos 
recém-nascidos - idade gestacional, peso ao nascer e Apgar no quinto minuto -, procedeuse à re-estratificação dos resultados controlados segundo a adequação ou não do número de consultas ao pré-natal.

Com base nesses dados - ressaltando-se que a variável tipo de parto acabou sendo desprezada diante da constatação da ausência de associação -, foram calculadas as razões de prevalência ajustadas 24 com os respectivos intervalos de confiança de $95 \%$, com vistas à consideração de diferenciais de risco, tendo por referência os valores observados entre as adultas com freqüência adequada ao pré-natal.

\section{Resultados}

A presença de mães adolescentes assistidas na rede hospitalar de Montes Claros foi da ordem de $21,5 \%$, sendo de $0,6 \%$ na faixa de 10 a 14 anos e $20,9 \%$ na faixa de 15 a 19 anos. Essas proporções, próximas às do país (23,4\% em 2000), pouco variaram no período de 1997 a 2001.

A taxa específica de fecundidade na adolescência entre as residentes em Montes Claros, no ano de 2001, por sua vez, foi de 3,84 por cem mulheres, sendo de 0,22 na faixa de 10 a 14 anos e de 7,02 na faixa de 15 a 19 anos. Essas mesmas taxas, no país, no ano 2000 , foram, respectivamente, 4,28 por cem mulheres, sendo de 0,33 na faixa de 10 a 14 e de 8,08 na faixa de 15 a 19 anos. Apresentando valores ligeiramente inferiores aos do país, as taxas específicas de fecundidade de Montes Claros, na faixa de 10 a 19 anos de idade, envolveram significativo crescimento. Levando em conta o período de 1997 a 2000 (para efeito de comparação), as taxas específicas de fecundidade, em Montes Claros, aumentaram de 2,44 para 3,73 (por cem mulheres), enquanto que, no país, esses valores passaram de 4,01 para 4,28.

A proporção de freqüência de pré-natal acima de seis consultas (Tabela 1) foi de $40,0 \%$ entre as adultas, sendo essa proporção significantemente menor entre as adolescentes, particularmente, na faixa de 10 a 14 anos. A proporção de realização de cesariana na população de parturientes adultas, por sua vez, foi de $35,0 \%$, enquanto que esse valor atinge $21,0 \%$ na faixa etária de 15 a 19 anos e, $20,0 \%$ na faixa de 10 a 14 anos.

Entre as adultas a ocorrência de prematuridade foi de 5,6\% no ano; essas proporções foram significantemente superiores na faixa de 15 a 19 anos e de 10 a 14 anos, tendo atingido, respectivamente, $7,4 \%$ e $25,5 \%$. A ocorrência de baixo peso, da ordem de $7,8 \%$ entre as partu- rientes adultas, chegou a $11,7 \%$ na faixa de 15 a 19 anos e, a $35,2 \%$ na faixa de 10 a 14 anos, sendo as diferenças significantes, levando em conta os respectivos intervalos de confiança. O Apgar do quinto minuto abaixo de 7 , da ordem de $1,95 \%$ entre as adultas, chegou a $3,3 \%$ na faixa de 15 a 19 anos de idade e atingiu $6,0 \%$ na adolescência precoce, ressaltando-se, entretanto, a sobreposição dos intervalos de confiança.

Considerando a freqüência ao pré-natal, no caso da prematuridade e baixo peso ao nascer, observamos (Tabela 2 e Tabela 3 ) número muito baixo de mães que apresentavam níveis adequados de consultas a esse serviço no segmento da adolescência precoce.

O pré-natal não-adequado conferiu uma chance aumentada de ocorrência de prematuridade e baixo peso ao nascer. Em que pese a proximidade dos valores das razões de prevalência entre as adultas $(2,38)$ e adolescentes de 15 a 19 anos $(2,80)$, chama a atenção a tendência de aumento no sentido das idades mais jovens, chegando a 7,41 vezes no caso da prematuridade e de 7,39 no caso do baixo peso, na faixa de 10 a 14 anos de idade.

Por outro lado, chama a atenção a ocorrência de prematuridade e baixo peso dos nascidos entre as mães com adequada freqüência ao pré-natal, registrando-se um distanciamento significante das razões de prevalência em relação à faixa de 10 a 14 anos.

As diferenças de risco entre mães com freqüência adequada e inadequada ao pré-natal, significativas para as faixas de 15 a 19 anos de idade e de 20 anos e mais, não puderam ser constatadas na faixa de 10 a 14 anos de idade, diante do pequeno número de casos e correspondente amplitude do intervalo de confiança.

Em relação ao Apgar de quinto minuto abaixo de 7 (Tabela 4), ao lado da pequena variação da intensidade dos valores da razão de prevalência, em que pese a ausência de casos dessas implicações entre adolescentes de 10 a 14 anos de idade com adequada freqüência ao pré-natal, observou-se superposição de intervalos de confiança entre as categorias consideradas.

\section{Discussão}

A proporção de partos de mães adolescentes na rede hospitalar de Montes Claros $(21,5 \%)$ se aproxima dos valores observados no Brasil, tendo por referência a proporção calculada para o ano $2000(23,4 \%)$. Esses valores são elevados em relação às proporções de outros países, ressalvadas as diferenças da natureza e ano de 
Freqüência de ocorrência de pré-natal adequado, cesáreas, prematuridade, baixo peso e índice de Apgar ao quinto minuto, com seus respectivos intervalos de confiança de 95\% (IC95\%), segundo grupo etário da mãe. Montes Claros, Minas Gerais, Brasil, 2001.

\begin{tabular}{|c|c|c|c|c|c|c|c|c|c|}
\hline \multirow[t]{3}{*}{ Variáveis } & \multicolumn{9}{|c|}{ Grupo etário da mãe (anos) } \\
\hline & \multicolumn{3}{|c|}{$10-14$} & \multicolumn{3}{|c|}{ 15-19 } & \multicolumn{3}{|c|}{20 e mais } \\
\hline & $\mathrm{n}$ & $\%$ & IC95\% & $n$ & $\%$ & IC95\% & $\mathrm{n}$ & $\%$ & IC95\% \\
\hline \multicolumn{10}{|c|}{ Pré-natal adequado } \\
\hline Sim & 6 & 12,0 & $3,0-21,0$ & 458 & 29,0 & $27,0-31,0$ & 2.354 & 40,0 & $38,8-41,3$ \\
\hline Não & 44 & 88,0 & $79,0-97,0$ & 1.119 & 71,0 & $68,7-73,2$ & 3.529 & 60,0 & $58,8-61,3$ \\
\hline Subtotal & 50 & 100,0 & & 1.577 & 100,0 & & 5.883 & 100,0 & \\
\hline \multicolumn{10}{|l|}{ Cesárea } \\
\hline Sim & 10 & 20,0 & $14,3-25,7$ & 336 & 21,0 & $20,0-22,1$ & 2.097 & 35,0 & $34,0-36,3$ \\
\hline Não & 40 & 80,0 & $74,8-85,7$ & 1.259 & 79,0 & $77,9-80,9$ & 3.877 & 65,0 & $63,7-66,1$ \\
\hline Subtotal & 50 & 100,0 & & 1.595 & 100,0 & & 5.974 & 100,0 & \\
\hline \multicolumn{10}{|l|}{ Prematuro } \\
\hline Sim & 13 & 25,5 & $19,4-31,6$ & 118 & 7,4 & $6,7-8,0$ & 333 & 5,6 & $5,3-5,9$ \\
\hline Não & 38 & 74,5 & $68,4-80,6$ & 1.469 & 92,6 & $92,0-93,3$ & 5.615 & 94,4 & $94,1-94,7$ \\
\hline Subtotal & 51 & 100,0 & & 1.587 & 100,0 & & 5.948 & 100,0 & \\
\hline \multicolumn{10}{|l|}{ Baixo peso } \\
\hline Sim & 18 & 35,2 & $22,2-48,4$ & 189 & 11,7 & $10,2-13,4$ & 469 & 7,8 & $7,1-8,5$ \\
\hline Não & 33 & 64,7 & $51,6-77,8$ & 1.415 & 88,2 & $86,6-89,8$ & 5.544 & 92,2 & $91,5-92,9$ \\
\hline Subtotal & 51 & 100,0 & & 1.604 & 100,0 & & 6.013 & 100,0 & \\
\hline \multicolumn{10}{|l|}{ Apgar $<7$} \\
\hline Sim & 3 & 6,0 & $0,6-12,3$ & 52 & 3,3 & $2,4-4,1$ & 109 & 2,0 & $1,5-2,1$ \\
\hline Não & 48 & 94,0 & $10,1-87,6$ & 1.548 & 96,7 & $95,9-97,6$ & 5.897 & 98,0 & $97,8-98,5$ \\
\hline Subtotal & 51 & 100,0 & & 1.600 & 100,0 & & 6.006 & 100,0 & \\
\hline Total & 51 & & & 1.605 & & & 6.016 & & \\
\hline
\end{tabular}

Fonte: Setor de Processamento de Dados, Secretaria Municipal de Saúde de Montes Claros, Minas Gerais, Brasil, 2001.

realização desses estudos, bem como a singularidade das respectivas estruturas populacionais, padrões e comportamentos reprodutivos locais - a proporção de partos de adolescentes foi de $0,9 \%$ na Holanda, $2,3 \%$ em Israel 15 , $12,8 \%$ nos Estados Unidos 17, 13,0\% na União Soviética e 14,4\% em Moçambique 13.

Em Montes Claros, as proporções observadas, num patamar mais elevado, condizem com expressivo rejuvenescimento da estrutura da fecundidade, que acompanha a abrupta queda da fecundidade no país em décadas recentes. O valor encontrado em 1999, da ordem de 21,4\%, atesta a tendência de aumento identificada no início do estudo, que apontava para um crescimento, no período de 1994 para 1996, de 9,1\% para $17,5 \%$.

Quanto à extensão de adolescentes que engravidaram e tiveram filhos nascidos vivos em
Montes Claros, embora as taxas específicas de fecundidade fossem ligeiramente inferiores, os valores encontrados, no município, consubstanciaram a hipótese de sua aproximação aos valores do país. Ressalta-se que tal aproximação envolveu um crescimento da ordem de $50,0 \%$ no período - a taxa específica de fecundidade, em Montes Claros, no ano de 1997, era de 2,44; passou para 3,73 no ano 2000, e alcançou 3,84 em 2001 - enquanto que, no país, esses valores se estabilizaram em torno de 4,00 nascimentos por cem mães adolescentes $(4,01$ em 1997 e 4,28 no ano 2000).

Vale dizer que, diferentemente do comportamento observado em Belo Horizonte, cujas taxas específicas de fecundidade apresentaram, no mesmo período, um decréscimo (de 3,39 em 1997 para 2,75 em 2001), os valores estimados para Montes Claros acompanharam de perto 
Tabela 2

Prevalência e razão de prevalência, com respectivo intervalo de confiança de 95\% (IC95\%), de nascidos vivos prematuros, segundo grupo etário da mãe e adequação do pré-natal. Montes Claros, Minas Gerais, Brasil, 2001.

\begin{tabular}{|c|c|c|c|c|c|c|}
\hline Idade (anos) & Pré-natal & Prematuro & Não prematuro & Prevalência & Razão de prevalência & IC95\% \\
\hline \multirow[t]{2}{*}{$10-14$} & Não-adequado & 10 & 33 & 0,230 & 7,41 & $4,11-13,34$ \\
\hline & Adequado & 2 & 4 & 0,330 & 10,64 & $3,35-33,66$ \\
\hline \multirow[t]{2}{*}{$15-19$} & Não-adequado & 97 & 1008 & 0,087 & 2,80 & $2,08-3,76$ \\
\hline & Adequado & 18 & 438 & 0,039 & 1,25 & $0,76-2,09$ \\
\hline \multirow[t]{2}{*}{20 e mais } & Não-adequado & 255 & 3179 & 0,074 & 2,38 & $1,83-3,05$ \\
\hline & Adequado & 73 & 2252 & 0,031 & 1,00 & $0,73-1,38$ \\
\hline
\end{tabular}

Fonte: Setor de Processamento de Dados, Secretaria Municipal de Saúde de Montes Claros, Minas Gerais, Brasil, 2001.

Tabela 3

Prevalência e razão de prevalência, com respectivo intervalo de confiança de 95\% (IC95\%), de nascidos vivos com baixo peso, segundo grupo etário da mãe e adequação do pré-natal. Montes Claros, Minas Gerais, Brasil, 2001.

\begin{tabular}{|c|c|c|c|c|c|c|}
\hline Idade (anos) & Pré-natal & Baixo peso & Não baixo peso & Prevalência & Razão de prevalência & IC95\% \\
\hline \multirow[t]{2}{*}{$10-14$} & Não-adequado & 17 & 27 & 0,380 & 7,39 & $4,90-11,14$ \\
\hline & Adequado & 1 & 5 & 0,200 & 3,84 & $0,53-19,24$ \\
\hline \multirow[t]{2}{*}{$15-19$} & Não-adequado & 152 & 967 & 0,135 & 2,59 & $2,07-3,26$ \\
\hline & Adequado & 33 & 424 & 0,072 & 1,38 & $0,95-2,00$ \\
\hline \multirow[t]{2}{*}{20 e mais } & Não-adequado & 338 & 3.189 & 0,095 & 1,82 & $1,50-2,24$ \\
\hline & Adequado & 123 & 2.230 & 0,052 & 1,00 & $0,78-1,28$ \\
\hline
\end{tabular}

Fonte: Setor de Processamento de Dados, Secretaria Municipal de Saúde de Montes Claros, Minas Gerais, Brasil, 2001.

Tabela 4

Prevalência e razão de prevalência, com respectivo intervalo de confiança de 95\% (IC95\%), de nascidos vivos com Apgar < 7 , grupos etários da mãe e adequação do pré-natal. Montes Claros, Minas Gerais, Brasil, 2001.

\begin{tabular}{|c|c|c|c|c|c|c|}
\hline Idade (anos) & Pré-natal & Apgar $<7$ & Apgar $\geq 7$ & Prevalência & Razão de prevalência & IC95\% \\
\hline \multirow[t]{2}{*}{$10-14$} & Não-adequado & 3 & 41 & 0,068 & 5,93 & $1,75-17,47$ \\
\hline & Adequado & 0 & 6 & 0,000 & 0,00 & - \\
\hline \multirow[t]{2}{*}{$15-19$} & Não-adequado & 39 & 1.075 & 0,035 & 2,84 & $1,76-4,57$ \\
\hline & Adequado & 10 & 448 & 0,021 & 1,75 & $0,87-3,61$ \\
\hline \multirow[t]{2}{*}{20 e mais } & Não-adequado & 78 & 3.445 & 0,022 & 1,83 & $1,18-2,74$ \\
\hline & Adequado & 29 & 2.322 & 0,012 & 1,00 & $0,60-1,67$ \\
\hline
\end{tabular}

Fonte: Setor de Processamento de Dados, Secretaria Municipal de Saúde de Montes Claros, Minas Gerais, Brasil, 2001. 
os valores do Estado de Minas Gerais, que passaram de 2,03 em 1997 para 3,30 em 2001.

$\mathrm{O}$ aumento das proporções da presença de mães adolescentes na rede hospitalar de Montes Claros, assim como das taxas específicas de fecundidade no município, coloca em relevo a consideração dos riscos relacionados à gravidez em idades precoces e condições associadas, como elementos significativos no dimensionamento da gravidez na adolescência como questão de saúde pública na região.

Os dados de freqüência ao pré-natal no estudo, acompanhando as proporções estimadas para o país, evidenciaram elevadas proporções de inadequação, que variaram segundo a idade. Ressalta-se, neste quadro, a presença maior de inadequação entre as adolescentes, como era esperado, não só pelos problemas de acesso, em geral, mas devido a dificuldades específicas observadas entre os adolescentes para a procura de serviços médicos, como o medo de procedimentos obstétricos, vergonha dos pais, assim como de abordagem sobre práticas sexuais, entre outras questões 11,16. Reafirma-se, nessas condições, a consideração do caráter interveniente dessa variável.

A proporção de cesariana entre as adolescentes, por sua vez, da ordem de $21,0 \%$, foi menor do que entre as adultas (ao redor de 35,0\%). Com valores condizentes com o selo de Hospital Amigo da Criança e de Maternidade Segura com os quais se qualificam as instituições da rede hospitalar de Montes Claros, esses dados evidenciaram a primazia do parto vaginal na adolescência, ressaltando-se, outrossim, a magnitude significativa da proporção de cesarianas nesse segmento etário. A propósito desses diferenciais, Lubarsky et al. 25, nos Estados Unidos, registraram $23,5 \%$ de cesáreas em adultas e 13,8\% entre adolescentes; Lao \& Ho 26, em Hong Kong, encontraram, respectivamente, nesses segmentos etários, $12,6 \%$ e 4,1\%; Mahfouz et al. 18 , por sua vez, encontraram $6,9 \%$ e $3,2 \%$.

Afastado, entretanto, o caráter epidêmico do recurso às cesáreas vigentes no país nos anos 90 , com registros superiores a 50,0\% e 75,0\% $6,27,28$, deixa de ter sentido a consideração do caráter interveniente dessa variável na consideração dos resultados perinatais, que fundamentava, originalmente, a estruturação do estudo. Permanecem, nestas condições, indagações sobre as possibilidades de redução dos atuais níveis de partos cirúrgicos, o que constituiria objeto de investigações específicas.

Dentre as variáveis desfecho, a ocorrência de prematuridade e baixo peso ao nascer, em Montes Claros, confirmaram maior presença nas idades mais precoces. Esses valores, exce- ção feita aos montantes dos percentuais encontrados na faixa de 10 a 14 anos, estariam muito próximos dos níveis estimados para o país no ano 2000, quando a prematuridade atingiu $6,63 \%$ entre parturientes adultas, $7,36 \%$ entre adolescentes de 15 a 19 anos de idade e 9,89\% entre 10 e 14 anos; no caso do baixo peso ao nascer, os valores estimados para o país variaram de 7,5 a 7,8\% no período de 1997 a 2001 para o conjunto das idades, observando-se a maior freqüência de baixo peso na faixa de idade de 10 a 14 anos, que alcançou 13,03\% em 1998.

Resguardadas as diferenças em relação à faixa de 10 a 14 anos, cuja variação poderia ser atribuída ao pequeno contingente identificado em Montes Claros, os resultados também apresentaram ordens de valores compatíveis com estudos realizados em outras localidades do país. Gama et al. 7 identificaram, no Rio de Janeiro, $7,3 \%$ de prematuridade na faixa de 15 a 19 anos de idade e 6,3\% entre as adultas de 20 a 24 anos, no período de 1996-1998. Num quadro de maior presença de prematuridade, Simões et al. 8 identificaram, em São Luiz, no ano de $1997,11,2 \%$ entre adultas de 20 a 24 anos e $22,9 \%$ na faixa de 13 a 17 anos, valores próximos aos de Montes Claros na faixa de 10 a 14 anos de idade.

Em relação ao baixo peso ao nascer, Gama et al. 7 encontraram $12,8 \%$ entre adolescentes de 15 a 19 anos, no Rio de Janeiro, e 9,1\% entre adultas de 20 a 24 anos de idade; Melo et al. 27 encontraram até $11,0 \%$ de baixo peso ao nascer, nas regiões administrativas do Estado de São Paulo, entre as adolescentes (sem distinguir a faixa de 10 a 14 e 15 a 19 anos) e uma variação de 8,0 a 9,0\% entre as adultas jovens. Bacci et al. 13, em Moçambique, num contexto socialmente diferenciado, encontraram valores superiores: $15,5 \%$ entre as adultas e $21,7 \%$, num total de 2.185 adolescentes.

No controverso equacionamento das complicações da gravidez na adolescência enquanto um problema associado à idade ou às condições assistenciais, Lubarsky et al. 25, assim como Berenson et al. 4, nos Estados Unidos (Texas e Tennessee), não encontraram diferenças de prematuridade e baixo peso ao nascer entre adultas e adolescentes. Griffiths 29 , por sua vez, ressaltando o caráter interveniente das condições assistenciais, encontrou relação significante entre a inadequação do pré-natal e a ocorrência de complicações do recém-nascido, principalmente de prematuridade e baixo peso ao nascer.

Evidenciando a relevância das condições assistenciais no tocante à prematuridade, Ramirez et al. 30 observaram que, tanto no grupo 
das adolescentes como no das adultas, só tiveram recém-nascidos prematuros as parturientes com pré-natal inadequado. A propósito, Fraser et al. 17 identificaram, entre pacientes adolescentes que não fizeram pré-natal, um risco relativo maior para a ocorrência de recémnascidos prematuros e de baixo peso, ressaltando que a ocorrência dessas complicações era elevada mesmo quando recebiam esse cuidado.

Em nosso meio, Gama et al. 7, em amostra hospitalar que excluía gemelares e mulheres com mais de 35 anos de idade, observaram, no período de 1996-1998, no Município do Rio de Janeiro, variações nas proporções de prematuridade em função da freqüência ao pré-natal, tendo constatado $8,8 \%$ de prematuridade entre adolescentes que freqüentaram sete e mais consultas; $14,5 \%$ entre aquelas que freqüentaram de quatro a seis consultas e $30,7 \%$ na faixa de zero a três consultas. Os mesmos autores, estudando a relação da adolescência com o baixo peso ao nascer, observaram um risco de 1,35 vezes maior na condição de um pré-natal inadequado e de 1,67 na ausência de pré-natal.

Na relação da prematuridade e do baixo peso com a idade, em nosso estudo, levando em conta o pré-natal, os cálculos das razões de prevalência evidenciaram elevada presença desses desfechos entre as parturientes que não freqüentaram adequadamente o pré-natal, sendo crescente o risco dessa ocorrência no sentido da idade adulta para as mais jovens - reafirmando, como era esperado, o peso do pré-natal no tocante a essa implicação.

Sem diminuir a relevância do pré-natal, vale considerar a elevação da chance de ocorrência de prematuridade e baixo peso ao nascer, inversamente associados com a idade, entre mães que apresentavam níveis adequados de freqüência ao pré-natal. Enquanto indício da existência de um risco precípuo da idade, nas condições sociais de existência da população de estudo, esses resultados seriam compatíveis com os achados de Fraser et al. 17, entre outros.

Levando em conta que as manifestações desses desfechos também se constituem na expressão das condições sociais de existência que circunscrevem a própria assistência, não se pode deixar de considerar que o reduzido número de mães na faixa de 10 a 14 anos de idade, com adequado pré-natal, comprometeu, em certo sentido, a comparação das razões de prevalência na adolescência precoce, limitando a consideração do peso específico do pré-natal como fator de proteção, no âmbito do presente estudo.

O índice de Apgar no quinto minuto também variou em função da idade materna $(2,9 \%$ entre as adultas, $3,3 \%$ na faixa de 15 a 19 anos e
$6,0 \%$ na faixa de 10 a 14 anos), ressaltando-se, contudo, a limitação na aceitação das diferenças devido à superposição de intervalos de confiança.

Num quadro de valores generalizadamente superiores, Lubarsky et al. 25 referem ter encontrado (nos Estados Unidos) 4,2\% de Apgar no quinto minuto abaixo de 7 entre as adultas e $5,4 \%$ no grupo de adolescentes, próximos, por conseguinte, aos de Montes Claros, na faixa de 10 a 14 anos, no ano de 2001 . Por outro lado, Gama et al. 7, no Rio de Janeiro, assim como Bacci et al. 13, Lao \& Ho 26, Berenson et al. 4, em outros países, não observaram diferenças na relação dessa variável e idade materna.

Controlada essa variável pela freqüência ao pré-natal, diferentemente do que aconteceu com a prematuridade e o baixo peso dos recémnascidos, não foram observadas diferenças significativas de razões de prevalência diante da superposição dos intervalos de confiança apresentados, tanto entre mães que apresentaram registro de número de consultas ao pré-natal adequado ou inadequado. Se o aumento do risco entre as mães com inadequada freqüência ao pré-natal não pode ser confirmado, tampouco caberia a consideração da existência de um risco intrínseco de exposição, para esse desfecho, associado à idade. Esses resultados guardam coerência com os achados de D’Orsi \& Carvalho 6 a propósito da distribuição espacial dos nascidos vivos, no Rio de Janeiro, em 1994. De acordo com os autores, os índices baixos de Apgar se concentraram nos bairros periféricos, assim como aconteceu com a distribuição de nascidos vivos de mães adolescentes, sugerindo estreita associação com as condições sócioeconômicas, ao contrário do baixo peso e da prematuridade, cuja distribuição ocorreu em todos os bairros.

\section{Considerações finais}

À guisa de finalização, vale dizer que a proporção de nascidos vivos de mães adolescentes evidencia a participação significativa desse contingente na rede hospitalar de Montes Claros, em 2001, ressaltando-se que esses valores concretizam um crescimento progressivo desencadeado na segunda metade dos anos 90 . Com valores superiores aos de Minas Gerais, as taxas específicas de fecundidade entre adolescentes de Montes Claros, por sua vez, sinalizaram para a aproximação aos valores do país, ressaltando-se, igualmente, um crescimento substantivo, considerado o espaço temporal dos últimos cinco anos. Para além das similitu- 
des de grandezas, esses resultados inscrevem a região no movimento de configuração dos padrões reprodutivos do país, que se estruturam a partir de meados dos anos 70, apontando para o aumento relativo e absoluto da gravidez na adolescência.

Em meio à generalizada proporção de limitada freqüência aos serviços de pré-natal, o estudo revelou maior presença de inadequação entre as adolescentes, particularmente expressiva na faixa de 10 a 14 anos de idade, descortinando a relevância da consideração das implicações da gravidez na adolescência em relação às condições de nascimento de seus filhos.

Com relação ao tipo de parto, o estudo evidenciou menor freqüência de cesárea entre as mais jovens. Afastado o caráter epidêmico de sua ocorrência, que pressupunha indicações injustificadas, os níveis superiores de sua ocorrência por referência ao de outros países sinalizariam para a necessidade de realização de estudos no sentido de qualificar a procedência de sua utilização.

Consubstanciando as suspeitas de maior freqüência destas implicações da gravidez na adolescência, tanto a prematuridade como o baixo peso do recém-nascido variaram segundo a idade, sendo mais freqüentes entre as adolescentes.

Controlados esses dados em função da freqüência ao pré-natal, as razões de prevalência indicaram variações significativas da chance de ocorrência desses desfechos nas faixas etárias consideradas, evidenciando que a freqüência adequada ao pré-natal, sem dúvida, confere proteção à ocorrência de complicações da gravidez, em geral, e na adolescência, em particular. Porém, a adequada freqüência a esse serviço não seria suficiente para garantir a ausência dessas complicações na gravidez precoce. Essas considerações em torno do caráter protetor da freqüência adequada ao pré-natal, de um lado, e dos indicativos da exposição “intrínseca" da idade às complicações relativas aos desfechos considerados, de outro, confirmaram a hipótese de concentração da presença de prematuridade e baixo peso ao nascer nas idades mais jovens e, em particular, na adolescência precoce.

Ressaltados os reduzidos níveis de freqüência adequada ao pré-natal na faixa de 10 a 14 anos de idade, tais resultados reafirmam a relevância da atenção ao adolescente como questão de saúde pública, não só diante da significativa presença desse segmento na rede hospitalar de Montes Claros, mas em vista dos indicativos da tendência de seu aumento no contexto demográfico regional, vislumbrando a mobilização que se faz necessária no sentido do atendimento às demandas locais e, mesmo, de reversão desse processo, como vem acontecendo em outras localidades do país.

\section{Resumo}

A presente investigação tem como propósito dimensionar a ocorrência de partos de adolescentes na rede hospitalar de Montes Claros, Minas Gerais, Brasil. Num universo de 7.672 Declarações de Nascidos Vivos do ano 2001, a proporção estimada de nascidos vivos de mães adolescentes foi de 21,5\%. Ao lado da reduzida presença de adequada freqüência ao pré-natal, particularmente na faixa de 10 a 14 anos de idade (12,0\%), o estudo apontou, no âmbito dos desfechos, para um crescimento da ocorrência de complicações, inversamente relacionada com a idade, sendo estas diferenças significativas no tocante à prematuridade e baixo peso ao nascer - o que não aconteceu com o Apgar no quinto minuto. Confirmando a hipótese de maior chance de ocorrência dessas complicações na vigência de um número inadequado de consultas ao pré-natal, os resultados também sinalizaram para a existência de um risco associado à idade, particularmente na adolescência precoce (10 a 14 anos). Ao lado dos indicativos de aumento da gravidez na adolescência, na região (período de 1997 a 2001), esses resultados apontaram para a consideração da gravidez na adolescência como problema de saúde pública.

Gravidez na Adolescência; Cuidado Pré-Natal; Idade Gestacional; Recém-Nascido de Baixo Peso; Índice de Apgar 


\section{Colaboradores}

P. Goldenberg foi responsável pela redação do artigo. M. C. T. Figueiredo participou do levantamento e tabulação dos dados. R. S. Silva colaborou na análise estatística.

\section{Referências}

1. Quijada JM, Vallenilla JM. Embarazo en la adolescencia - aspectos psicológicos y sociales. Arch Venez Psiquiatr Neurol 1987; 33:11-5.

2. Beretta MIR, Denari FE, Pedrazzani JC. Estudo sobre a incidência de partos na adolescência em um município do Estado de São Paulo. Rev Latino-Am Enfermagem 1995; 3:181-91.

3. Fujimori E, Oliveira IMV, Lima AR, Cassana LMN, Szarfac SC. Perfil socioeconómico y biológico de embarazadas adolescentes de una maternidad de beneficiencia en São Paulo, Brasil. Cuad Med Soc 1997; 38:97-114.

4. Berenson AB, Wiemann CM, McCombs SL. Adverse perinatal outcomes in young adolescents. J Reprod Med 1997; 42:559-63.

5. Halbe HW, Halbe AFP, Ramos LO. A saúde na adolescência: concepção do ginecólogo. In: Halbe HW, organizador. Tratado de ginecologia. São Paulo: Roca; 2000. p. 87-94.

6. D'Orsi E, Carvalho MS. Perfil de nascimentos no Município do Rio de Janeiro: uma análise espacial. Cad Saúde Pública 1998; 14:367-79.

7. Gama SGN, Szwacwald CL, Leal MDC, Theme Filha MM. Gravidez na adolescência como fator de risco para baixo peso ao nascer no Município do Rio de Janeiro, 1996 a 1998. Rev Saúde Pública 2001; 35:74-80.

8. Simões VMF, Bettio Filho H, Tonial SR, Mochel EG. Características da gravidez na adolescência em São Luiz, Maranhão. Rev Saúde Pública 2003; 37:529-65.

9. Daadorian D. A gravidez e o desejo na adolescência. Femina 2002; 30:133-4.

10. Organización Mundial de la Salud. Necesidades de salud de los adolescentes. Geneva: Organización Mundial de la Salud; 1977. (Série de Informes Técnicos n. 609).

11. Correa MD. Noções práticas de obstetrícia. 12a Ed. Minas Gerais: Medsi; 1999.

12. Amini SB, Catalano P, Dierker LR, Mann LI. Births to teenagers: trends and obstetric outcomes. Obstet Gynecol 1996; 87:668-73.

13. Bacci A, Manhica GM, Machungo F, Bugalho A, Cuttini M. Outcome of teenage pregnancy in Maputo, Mozambique. Int J Gynaecol Obstet 1993; 40:19-23.

14. Motta ML, Pinto e Silva JL. Gravidez entre adolescentes muito jovens. Femina 1994; 22:348-54.

15. Barda G, Arbel-Alon S, Bernstein D, Zakut H, Menezer J. Pregnancy and delivery in a group of Israeli teenagers. A case-controlled study. Clin Exp Obstet Gynecol 1998; 25:32-4.

16. Orvos H, Nyirati I, Hajdú J, Pal A, Nyári T, Kovács L. Is adolescent pregnancy associated with adverse perinatal outcome? J Perinat Med 1999; 27:199-203.
17. Fraser AM, Brockert JE, Ward RH. Association of young maternal age with adverse reproductive outcome. N Engl J Med 1995; 332:1113-7.

18. Mahfouz AAR, El-Said MM, Al-Erian RAG, Hamid AM. Teenage pregnancy: are a high risk group? Eur J Obstet Gynecol Reprod Biol 1995; 59:17-20.

19. Bukulmez O, Deren O. Perinatal outcome in adolescent pregnancies: a case - control study from a Turkish University Hospital. Eur J Obstet Gynecol Reprod Biol 2000; 88:207-12.

20. Gama SGN, Szwarcwald CL, Leal MC. Experiência de gravidez na adolescência, fatores associados e resultados perinatais entre puérperas de baixa renda. Cad Saúde Pública 2002; 18:153-61.

21. Melo AV. Gravidez na adolescência: uma nova tendência na transição da fecundidade no Brasil. In: Anais do X Encontro Brasileiro de Estudos Populacionais -20 anos de ABEP. Rio de Janeiro: Associação Brasileira de Estudos Populacionais; 1996. p. 1439-54.

22. Monteiro DLM, Cunha AA. Avaliação da freqüência da gravidez na adolescência. J Bras Ginecol $1994 ; 104: 23-5$.

23. Organização Mundial da Saúde. Saúde reprodutiva de adolescentes: uma estratégia para ação. Brasília: Organização Mundial da Saúde/Fundo das Nações Unidas para a População/Fundo das Nações Unidas para a Infância; 1989.

24. Thompson M, Meyers J, Kriebel D. Prevalence odds ratio or prevalence ratio in analysis of cross sectional data: what is to be done? Occup Environ Med 1998; 55:272-7.

25. Lubarsky SL, Schiff E, Friedman SA, Mercer BM, Sibai BM. Obstetric characteristics among nulliparas under age 15. Obstet Gynecol 1994; 84:365-8.

26. Lao TT, Ho LF. Obstetric outcome of teenage pregnancies. Human Reprod 1997; 13:3228-32.

27. Melo AV, Yazaki LM, Morell MGG. Risco e ônus das gestações precoces. In: SEADE - Estudo sóciodemográfico sobre a juventude paulista. São Paulo: Fundação Sistema Estadual de Análise de Dados; 1998. p. 151-7.

28. Moraes MS, Goldenberg P. Cesáreas: um perfil epidêmico. Cad Saúde Pública 2001; 17:509-19.

29. Griffithis EA. Embarazo en adolescentes: resultados de una intervencion en el nivel primario de salud. Cuad Med Soc 1995; 36:12-8.

30. Ramírez AT, Soto ILC, Zambrana MM, Ricalde RL. La resolución obstétrica de las adolescentes en comparación con las adultas. Ginecol Obstet Mex 1999; 67:377-84.

Recebido em 04/Jun/2004

Versão final reapresentada em 05/Nov/2004 Aprovado em 09/Dez/2004 